\title{
Seebeck Coefficient of Ultrathin Silicon-on-Insulator Layers
}

Faiz Salleh, Kiyosumi Asai, Akihiro Ishida ${ }^{1}$, and Hiroya Ikeda*

Research Institute of Electronics, Shizuoka University, 3-5-1 Johoku, Naka-ku, Hamamatsu 432-8011, Japan

${ }^{1}$ Department of Electrical and Electronic Engineering, Shizuoka University, 3-5-1 Johoku, Naka-ku, Hamamatsu 432-8561, Japan

\begin{abstract}
We measured the Seebeck coefficient of P-doped ultrathin silicon-on-insulator (SOI) layers with thicknesses of 2-100 $\mathrm{nm}$. The dependence of the coefficient on the impurity concentration was investigated, and was shown to be in good agreement with that of bulk Si for SOI thicknesses above 6 $\mathrm{nm}$. In addition, it was found to decrease with increasing impurity concentration, which is usually observed in semiconductor materials. However, for doping levels above $3.5 \times 10^{19} \mathrm{~cm}^{-3}$, the Seebeck coefficient was observed to increase. This is likely to be due to the influence of an impurity band.
\end{abstract}

In recent years, thermoelectric devices have attracted considerable attention due to their ability to produce electric power from waste heat and as a means of tackling issues related to global warming problem. In addition, it is expected that such devices can be utilized in noiseless, vibration-free refrigerators with zero greenhouse gas emission. However, the thermoelectric efficiency is still not enough for practical use. Nanostructure has been introduced to overcome this problem since the quantum confinement effect can enhance the thermoelectric efficiency. ${ }^{1,2)}$ In the present paper, we investigate the Seebeck coefficient $S$ of P-doped ultrathin silicon-on-insulator (SOI) layers in order to clarify the quantum confinement effect on Seebeck coefficient. The Si nanostructure is easy to be fabricated precisely and the SOI thermoelectric material can be used as a refrigeration system for Si-based devices such as central processing units (CPUs) or field emission displays (FEDs).

A schematic diagram of our experimental setup is shown in Fig. 1. Two gilded Cu-plates were placed side by side with a gap of $1 \mathrm{~mm}$ between them. Resistive heaters were placed beneath the plates and could be heated individually. The sample for measuring was placed across the gap, in contact with both of the plates. Therefore, by controlling the heater current, a temperature difference could be produced in a plane parallel to the sample surface. A couple of probes and two K-type thermocouples were directly attached to the sample surface. The time evolution of the thermoelectromotive force was measured by a digital multimeter (KEITHLEY 2700) equipped with a switching module (KEITHLEY 7700), simultaneously

\footnotetext{
*E-mail address: ikeda@rie.shizuoka.ac.jp
} 


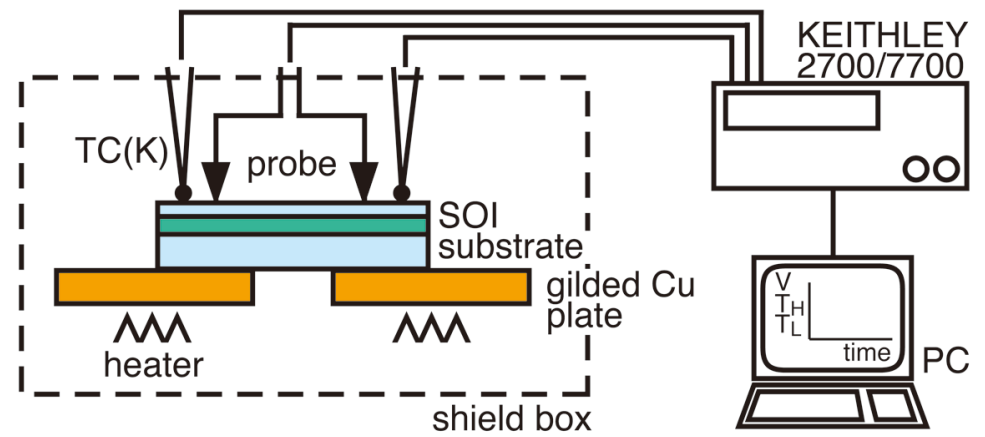

Fig. 1. Schematic diagram of the apparatus for Seebeck coefficient measurement.

with the temperatures at the high- and low-temperature regions. The Seebeck coefficient was evaluated from the thermoelectricmotive force $\left(\Delta V \equiv V_{H}-V_{L}\right)$ and the temperature difference $\left(\Delta T \equiv T_{H}-T_{L}\right)$ by $S=\Delta V / \Delta T$.

The SOI wafer consisted of a top Si layer (SOI layer), a buried oxide (BOX) layer and a p-type Si substrate, and it was cut to a size of $10 \times 10 \mathrm{~mm}^{2}$. The SOI layer was thinned to a thickness of 2 to $100 \mathrm{~nm}$ by repeated thermal oxidation and HF etching. $\mathrm{P}$ atoms were doped into the SOI layer by thermal diffusion to produce an n-type Si layer. The impurity concentration ranged from $2 \times 10^{17}$ to $5 \times 10^{19} \mathrm{~cm}^{-3}$, determined by a four-probe method at room temperature. The thickness of the BOX layer was $400 \mathrm{~nm}$ and the impurity concentration of the p-type Si substrate was $\sim 10^{16} \mathrm{~cm}^{-3}$.

The absolute value of the measured Seebeck coefficient of the SOI layers is shown in Fig. 2, as a function of impurity concentration. The numbers adjacent to the red filled-circles indicate the SOI layer thickness. Unfortunately, the SOI samples with a thickness of 2-4 nm could not be measured due to a large noise, so that only the Seebeck coefficients for the SOI samples above $6 \mathrm{~nm}$ thick are shown in Fig. 2. In this figure, the results for n-type Si wafers obtained from our measurements and reported in the literature ${ }^{3-5)}$ are also shown. The solid line is the theoretical Seebeck coefficient obtained from our calculations based on the electron transport. ${ }^{6}$ ) From Fig. 2, the values of the Seebeck coefficient appear to lie on a curved line (indicated by a broken line in Fig. 2) and to be independent of the SOI layer thickness. Therefore, the quantum confinement effect can not be observed above $6 \mathrm{~nm}$ in SOI thickness. In addition, the values for bulk Si wafers are very similar to those for SOI layers, which implies that a Si film as thin as $6 \mathrm{~nm}$ has the same Seebeck coefficient as a Si wafer. Hence, this fact suggests that an ultrathin Si film with a nanometer-scale thickness can replace the bulk $\mathrm{Si}$ thermoelectric material.

Below $2 \times 10^{19} \mathrm{~cm}^{-3}$, the absolute value of the experimental Seebeck coefficient of SOI layers decreases with an increase in the impurity concentration, as is generally seen in semiconductor 


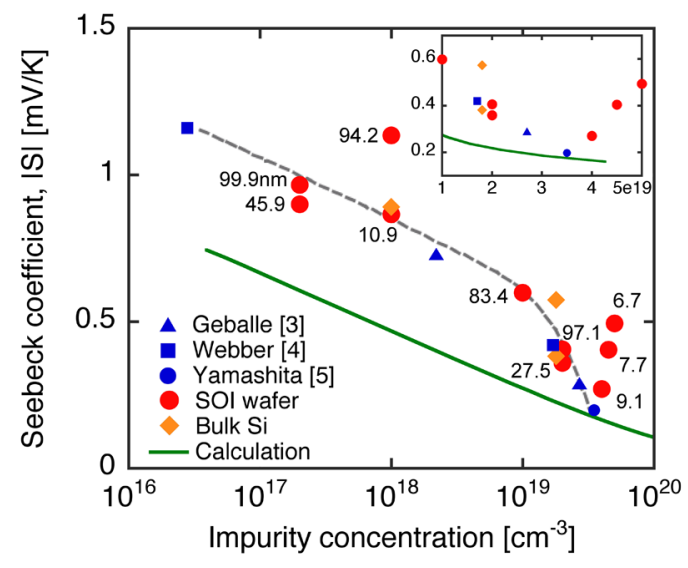

Fig. 2. Absolute Seebeck coefficient of SOI wafers as a function of impurity concentration. The Seebeck coefficients of $\mathrm{Si}$ wafers obtained from our measurements and reported in the literatures ${ }^{344) 5)}$ are also shown. The solid line represents the calculated value and the broken line is an eye-guide.

materials. ${ }^{7)}$ However, as seen in the figure, the theoretical curve does not fit well to the experimental data. This is considered to be due to the influence of phonon drag originating from momentum transfer from the phonon system to the electron system by electron-phonon scattering. ${ }^{4)}$ The influence of such phonon drag is known to become weak at high impurity concentration. ${ }^{4)}$

On the other hand, SOI layers with impurity concentrations above $3.5 \times 10^{19} \mathrm{~cm}^{-3}$ are found to exhibit unusual behavior in their Seebeck coefficients. The inset in Fig. 2 shows a magnified graph of this region. It is clearly seen that the absolute value of Seebeck coefficient increases with increasing impurity concentration. Since phonon drag is no longer significant at these impurity concentrations, ${ }^{4)}$ additional factors such as the influence of an impurity band must be considered. ${ }^{8)}$

In order to confirm the influence of an impurity band, the density of states (DOS) was calculated for highly-doped bulk Si, based on a Baltensperger model. ${ }^{9)}$ This model assumes a regular close-packed lattice built of hydrogen-like impurities where the Schrödinger equation is solved inside a sphere occupied by an impurity atom. The impurity band width $\Delta E$ is defined as the energy difference between the band edges obtained from boundary conditions for the wave functions. After obtaining the band width, a Gaussian curve is used to represent the actual DOS across the impurity band, with the maximum lying at the energy level corresponding to the original impurity level. ${ }^{10}$ ) We can express the impurity concentration $N_{D}$ as

$$
\frac{\Delta E N_{D}}{2 \sqrt{2 \pi} \sigma}=N_{D}
$$


where $\sigma$ is a parameter characterizing the extent of the Gaussian function. This expression corresponds to assuming that the Gaussian function has a triangular shape with a base of $\Delta E$ and a height of $N_{D} / \sqrt{2 \pi} \sigma$. The DOS function for the impurity band can then be represented by

$$
\rho_{i}(E)=\frac{2 N_{D}}{\Delta E} \exp \left\{-4 \pi\left(\frac{E-E_{D}}{\Delta E}\right)^{2}\right\},
$$

where $E_{D}$ is the donor level and the conduction band edge $E_{C}$ is set to zero.

The wave function includes the confluent hypergeometric series $F\left(l+1-n, 2 l+2 ; 2 r_{s} / n a_{B}\right)$ in the hydrogen-like model, ${ }^{9)}$ where $a_{B}$ is the Bohr radius and $r_{s}$ is the mean radius of the sphere occupied by an impurity atom. The indices $n$ and $l$ are the principal and the orbital quantum numbers, respectively. In this paper, the impurity band width is computed under the condition that the principal quantum number $n$ is approximately equal to unity for $l=0$, which corresponds to a 1s band. ${ }^{11)}$ Figure $3\left(\right.$ a) shows the calculated DOS for $N_{D}=5 \times 10^{17}$, $5 \times 10^{18}$ and $5 \times 10^{19} \mathrm{~cm}^{-3}$, in the case of $\mathrm{P}$ atoms $\left(E_{D}=-44 \mathrm{meV}\right)$. The Fermi energy for $N_{D}=5 \times 10^{19} \mathrm{~cm}^{-3}$, evaluated from the charge-neutrality condition, is also indicated by an arrow. Below $5 \times 10^{18} \mathrm{~cm}^{-3}$, it is likely that the DOS overlap between the impurity band and the conduction band is very small. On the other hand, for $N_{D}=5 \times 10^{19} \mathrm{~cm}^{-3}$, the DOS overlap becomes significant and the Fermi energy lies near the conduction band edge $E_{C}$. The impurity band undoubtedly influences the Seebeck coefficient at higher impurity concentration since the Seebeck coefficient is strongly dependent upon the DOS distribution around the Fermi energy. ${ }^{12)}$

The calculated Fermi energy and the impurity-band DOS at $E_{C}$ are shown in Fig. 3(b) as a function of impurity concentration. The values of $E_{D}$ for $\mathrm{P}$ atoms and $E_{C}$ are also shown in this figure. It is clearly seen that the Fermi energy rises with increasing impurity concentration and crosses the maximum of the impurity band DOS $\left(E_{D}\right)$ at $N_{D} \sim 1 \times 10^{19} \mathrm{~cm}^{-3}$. In addition, the impurity band DOS at an energy of $E_{C}$ also abruptly increases above $1 \times 10^{19} \mathrm{~cm}^{-3}$. These facts indicate that above $\sim 1 \times 10^{19} \mathrm{~cm}^{-3}$, a continuous distribution in DOS is produced by the overlap between the impurity and the conduction bands, and the Fermi level is located within this continuous band. The range of impurity concentrations where the DOS distribution drastically changes is in good agreement with the region of abrupt increase in the Seebeck coefficient shown in Fig. 2. Consequently, the complicated band structure in heavily doped $\mathrm{Si}$, which is quite different from that in weakly doped $\mathrm{Si}$, may be the origin of the Seebeck coefficient enhancement at higher impurity concentration.

Seeing the result of Fig. 2 from a different angle, it is possible that the relationship between the Seebeck coefficient and the logarithmic impurity concentration makes a straight line for the whole concentration range and there is an interesting phenomenon which lowers the Seebeck coefficient (e.g., it cancels the phonon-drag effect) only at $1 \times 10^{19} \sim 5 \times 10^{19} \mathrm{~cm}^{-3}$. However, 
(a)

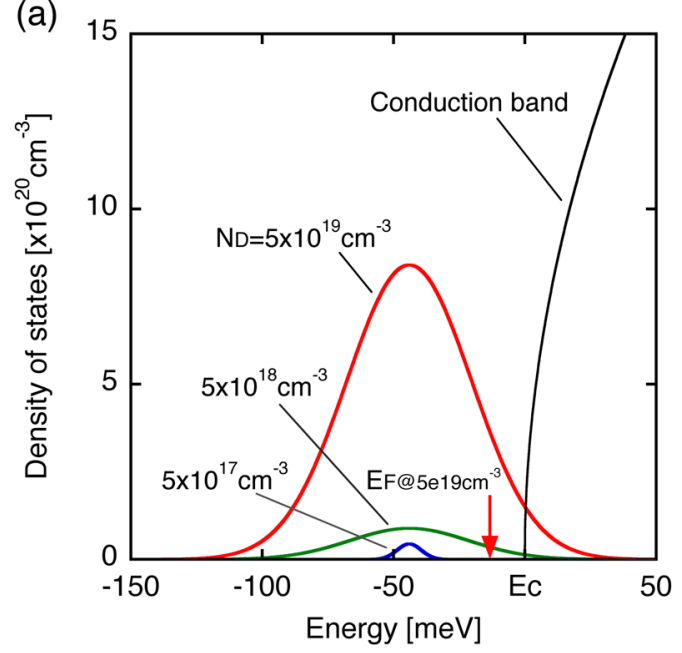

(b)

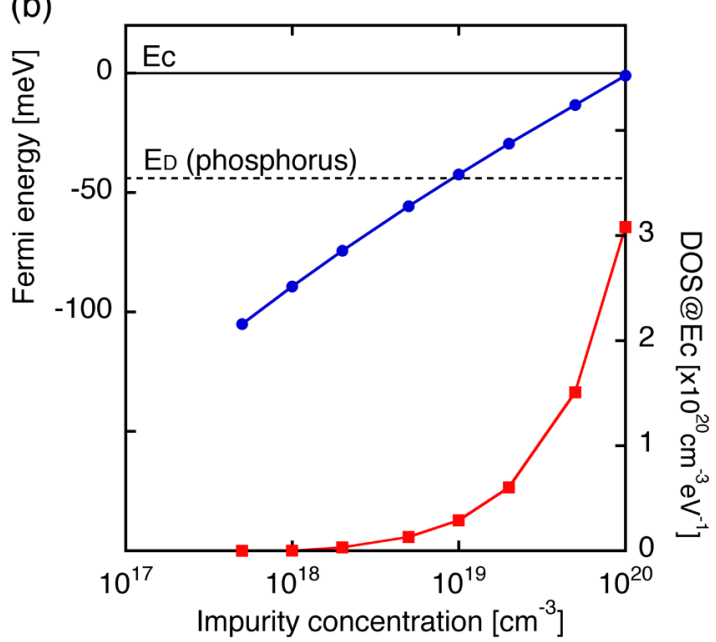

Fig. 3. (a) Density of states calculated for an impurity concentration of $N_{D}=5 \times 10^{17}, 5 \times 10^{18}$ and $5 \times 10^{19} \mathrm{~cm}^{-3}$, based on the Baltensperger model, and (b) calculated Fermi energy (•) and impurityband DOS at the conduction-band edge ( $)$ as a function of impurity concentration. The ionization energy is set to $E_{D}=44 \mathrm{meV}$ for phosphorus atoms and the Fermi energy is evaluated from the charge-neutrality condition.

there is not any idea about such phenomenon, so that we attribute the result of Fig. 2 to the influence of the phonon drag and the impurity band, as mention above. Moreover, the discussion on heavily doped samples is needed. Although we discussed only the impurity band in this paper, phenomena in a degenerated semiconductor such as conduction band tailing ${ }^{13)}$ also should be considered. From Fig. 3(a), the band structure is similar to that in Kondo semimetals and semiconductors. ${ }^{14)}$ Therefore, the Seebeck coefficient enhancement at higher impurity concentration may be characterized by a model of the thermoelectric material with a pseudogap. An analysis about the Seebeck coefficient at higher concentration needs further investigation.

We have investigated the Seebeck coefficient of SOI layers with thicknesses of 2-100 nm and found that it is very similar to that of bulk Si for SOI thicknesses above $6 \mathrm{~nm}$. This indicates that the quantum confinement effect is not observed in a Si layer as thin as $6 \mathrm{~nm}$. An enhancement of the Seebeck coefficient was observed at higher impurity concentrations $\left(N_{D}>1 \times 10^{19} \mathrm{~cm}^{-3}\right)$. This phenomenon is believed to be related to the formation of an impurity-band, and based on calculations of the impurity-band DOS, its influence is likely to be significant above $\sim 1 \times 10^{19} \mathrm{~cm}^{-3}$, which is consistent with the experimental results.

\section{Acknowledgement}

This work was financially supported by a Grant-in-Aid for Scientific Research (19560701 
and 21360336) from the Japan Society for the Promotion of Science. 


\section{References}

1) L. D. Hicks, T. C. Harman, X. Sun, and M. S. Dresselhaus: Phys. Rev. B 47 (1993) 12727.

2) L. D. Hicks and M. S. Dresselhaus: Phys. Rev. B 47 (1993) 16631.

3) T. H. Geballe and G. W. Hull: Phys. Rev. 98 (1955) 940.

4) L. Weber and E. Gmelin: Appl. Phys. A 53 (1991) 136.

5) O. Yamashita: J. Appl. Phys. 95 (2004) 178.

6) A. Ishida, D Cao, S. Morioka, Y. Inoue, and T. Kita: J. Electron. Mater. Published online, 04 March (2009).

7) G. D. Mahan: Solid State Phys. 51 (1979) 81.

8) J. P. Heremans, V. Jovovic, E. S. Toberer, A. Saramat, K. Kurosaki, A. Charoenphakdee, S. Yamanaka, and G. J. Snyder: Science 321 (2008) 554.

9) W. Baltensperger: Philos. Mag. 44 (1953) 1355.

10) T. P. Brody: J. Appl. Phys. 33 (1962) 100.

11) C. Erginsoy: Phys. Rev. 88 (1952) 893.

12) N. F. Mott and E. A. Davis: Electronic Processes in Non-Crystalline Materials (Clarendon Press, Oxford, 1979) P. 52.

13) D. S. Lee and J. G. Fossum: IEEE Trans. Electron Devices ED-30 (1983) 626.

14) T. Takabatake, T. Sasakawa, J. Kitagawa, T. Suemitsu, Y. Echizen, K. Umeo, M. Sera, and Y. Bando: Physica B 328 (2003) 53. 